\title{
Experimental results on heavy quark fragmentation
}

\author{
Leonid Gladilin \\ (SINP MSU)
}

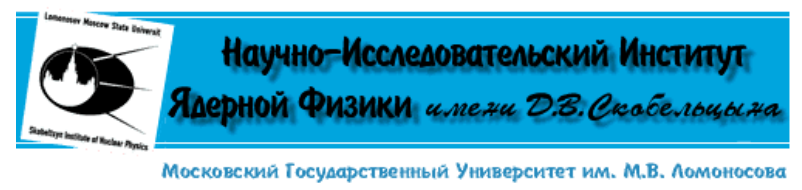

DIS 2006, April 20-24

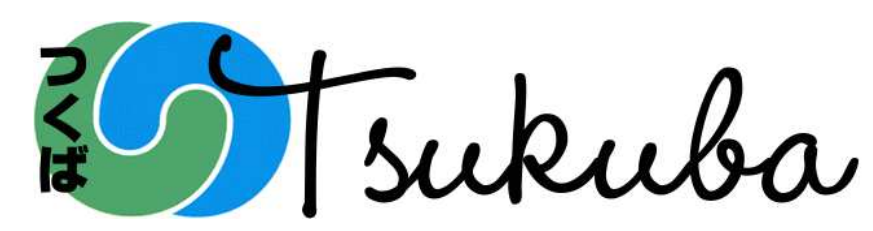

\section{O U T L I N E :}

Introduction

$b$ fragmentation functions

$b$ fragmentation branchings

$b$ fragmentation ratios

$c$ fragmentation in $e^{+} e^{-}$

$c$ fragmentation at HERA

B A C K U P :

Summary

estimates of extrapolation factors

fragm. branchings for excited $D$ mesons 


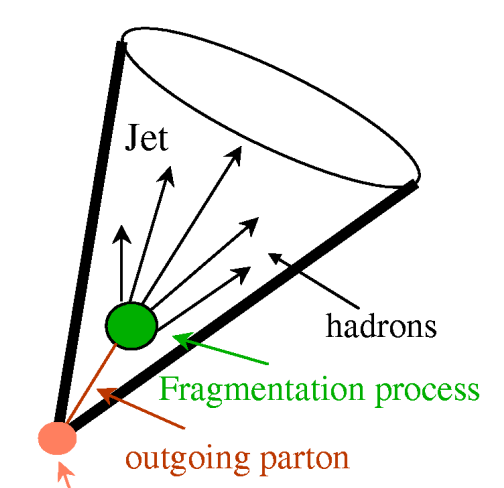

\section{Heavy Quark fragmentation issues}

\section{Important to measure}

HQ fragmentation to find :

Hard scatter

1) What is the proper parameterisation for the fractional transfer of $b / c$-quark energy/momentum to a given $B / D$-meson $(\mathbf{z})$ ? fragmentation function $(\mathbf{F F}), f(z)$ or $D(z)$

2) What are the relative fragm. branchings (FB's) of $B / D$-hadrons ? $f(c \rightarrow D)=\frac{N(D)}{N(c)}=\frac{\sigma(D)}{\sum_{\text {all }} \sigma(D)}$

a) Are $u$ and $d$ quarks produced equally ? $\quad R_{u / d}=\frac{c \bar{u}}{c \bar{d}}$

b) What is the $s$-quark production suppression ? $\quad \gamma_{s}=\frac{2 c \bar{s}}{c \bar{d}+c \bar{u}}$

c) Are vector $\left(B^{*} / D^{*}\right)$ and pseudoscalar $(B / D)$ mesons produced as predicted by spin counting ? $\quad P_{\mathrm{v}}=\frac{V}{V+P S} \quad(=0.75 ?)$

3) Are these functions, branchings and ratios universal ? compare results in $e^{+} e^{-}$annihilations with those at HERA 


\section{Heavy Quark fragmentation in $e^{+} e^{-}$annihilations}

\section{Production Fragmentation Decays}

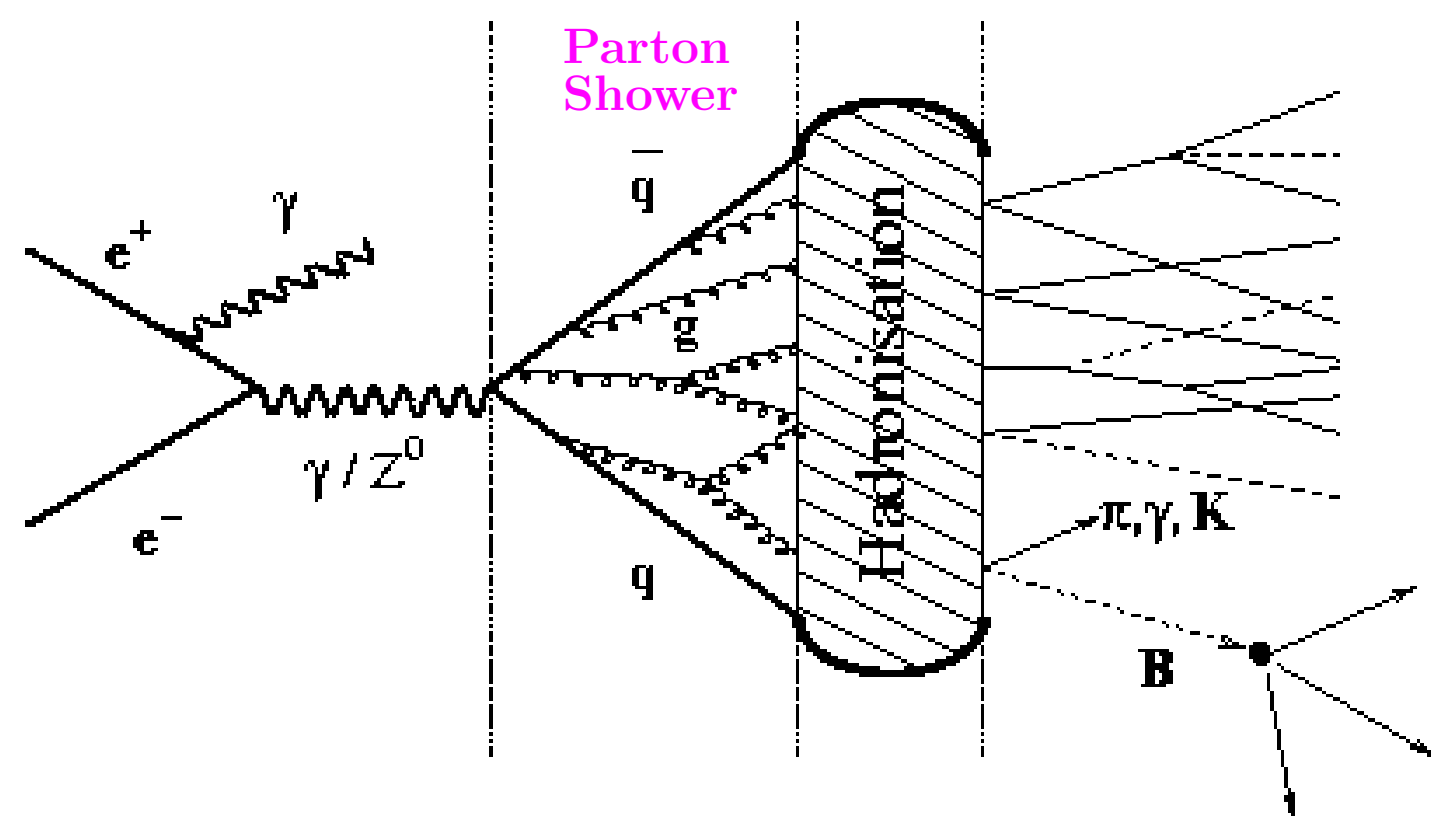

HQ fragmentation is hard harder for larger $m_{Q}$

e.g., for Peterson param.:

$f(z) \propto \frac{1}{z(1-1 / z-\epsilon /(1-z))^{2}}$

$\epsilon(b) \sim \frac{m_{c}^{2}}{m_{b}^{2}} \epsilon(c) \sim 0.1 \epsilon(c)$

pQCD is applicable to "initial" Q-fragmentation: LO, NLO, LL, NLL, ... anyhow, some parameterisation is needed for the non-perturbative (NP) rest the NP parameterisation is strongly dependent from the perturbative core (it is wrong to use MC fragmentation for $\mathrm{NLO}$ w/o full retuning the fragm. parameters) the NP parameterisation can include some decays FB's are expected to be independent from the perturbative core 


\section{$b$ fragmentation function, FF with LL MC}

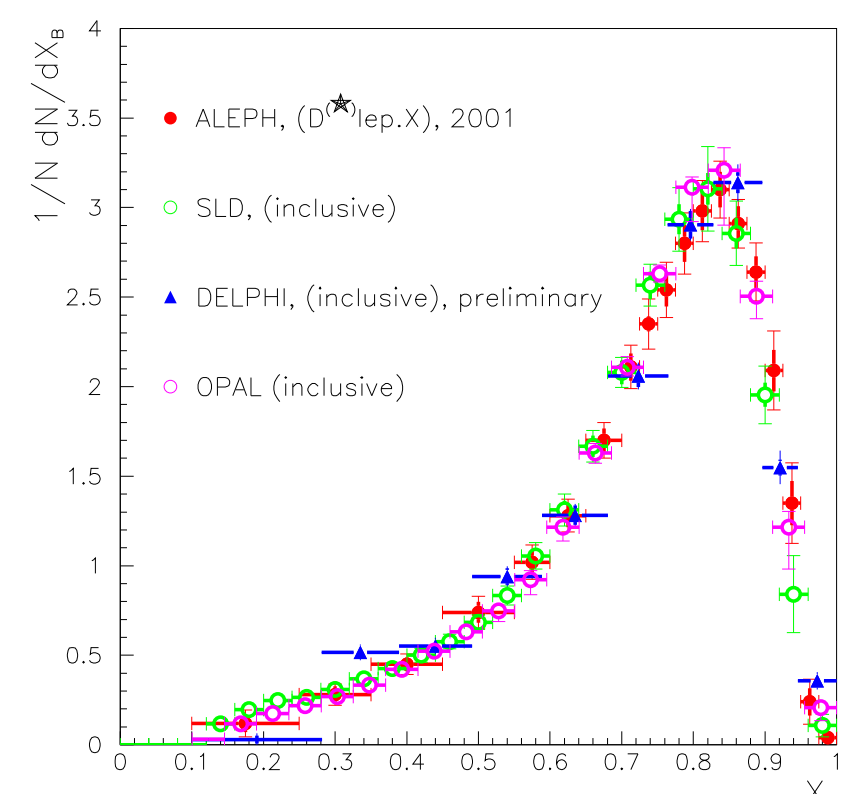

measured at LEP and SLD

from sec. vertices or $\mathrm{s} / \mathrm{l}$ decays (ALEPH)

in terms of the scaled energy: $x \equiv E_{\text {hadron }} / E_{\text {beam }}$ for weakly decaying $B$ hadrons

$<x>=0.7193 \pm 0.0016_{-0.0033}^{+0.0038}($ OPAL)

\section{FF with LL Monte Carlo}

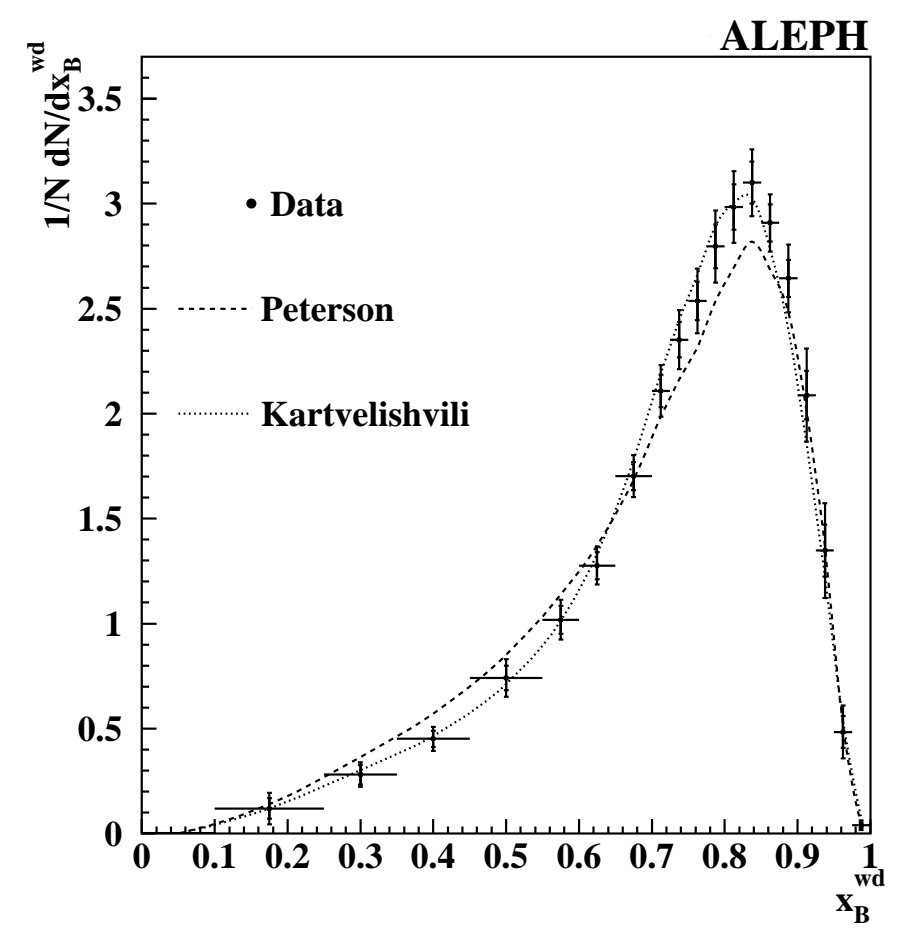

Bowler, $\frac{1}{z^{1+b m_{\perp}^{2}}}(1-z)^{a} \exp \left(\frac{-b m_{\perp}^{2}}{z}\right)$, and

Lund symmetric, $\frac{1}{z}(1-z)^{a} \exp \left(\frac{-b m_{\perp}^{2}}{z}\right)$, are

in good agreement with data (2 parameters)

Kartvelishvili et al., $z^{\alpha}(1-z)$, is o.k. with 1 parameter

Collins-Spiller, $\left(\frac{1-z}{z}+\frac{(2-z) \epsilon}{1-z}\right)\left(1+z^{2}\right)\left(1-\frac{1}{z}-\frac{\epsilon}{1-z}\right)^{-2}$, and

Peterson are too broad (1 par.)

$$
z \equiv\left(E+p_{\|}\right)_{\text {hadron }} /(E+p)_{\text {quark }}
$$

HERWIG cluster model disfavoured 


\section{$b$ FF with NLO+NLL+Sudakov pQCD}

Cacciari, Nason, Oleari
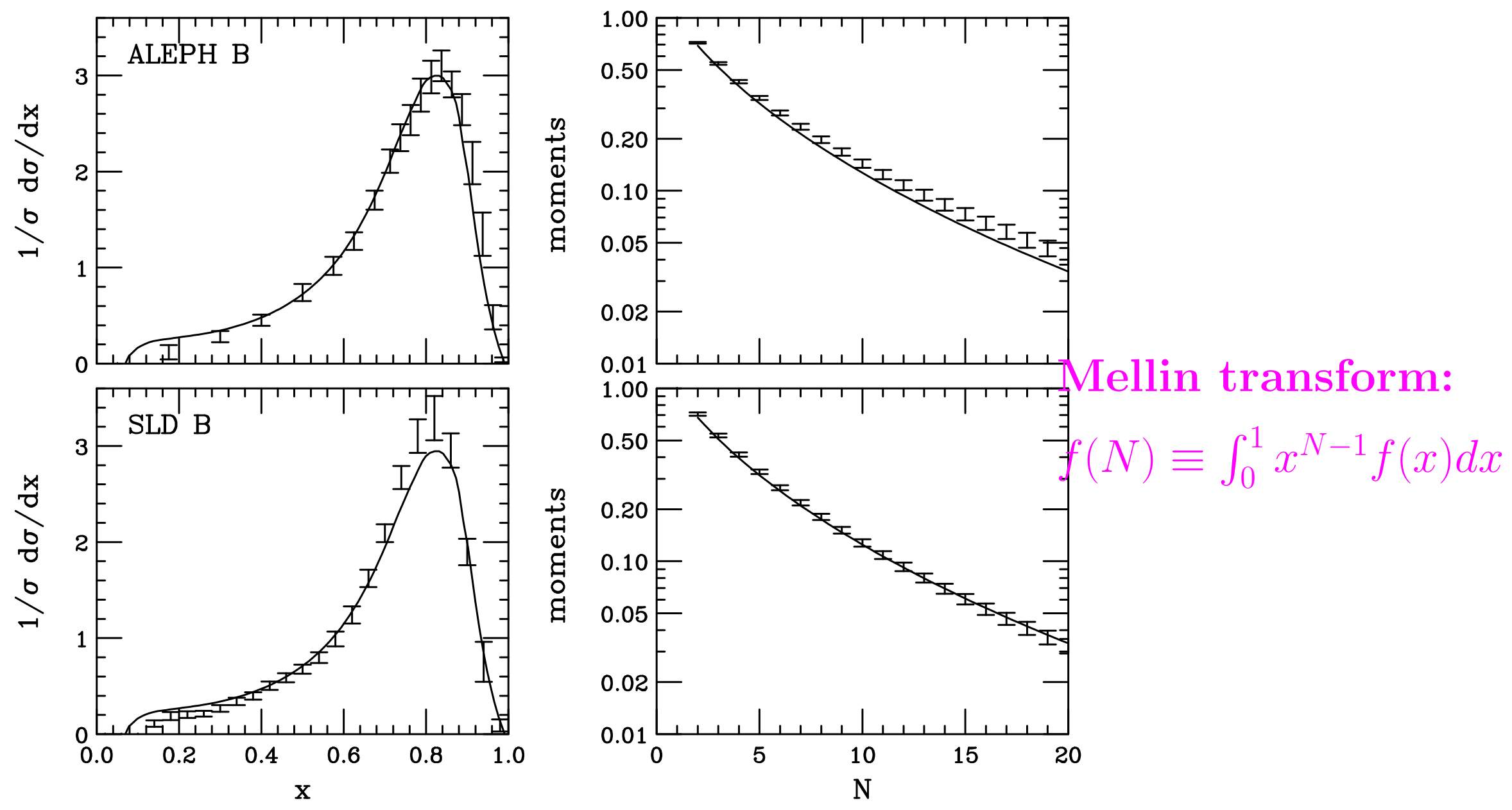

$D_{N P}(x) \propto(1-x)^{a} x^{b}$ (Colangelo-Nason)

provides reasonable description with $a=24 \pm 2, b=1.5 \pm 0.2$ 


\section{$b$ fragmentation branchings}

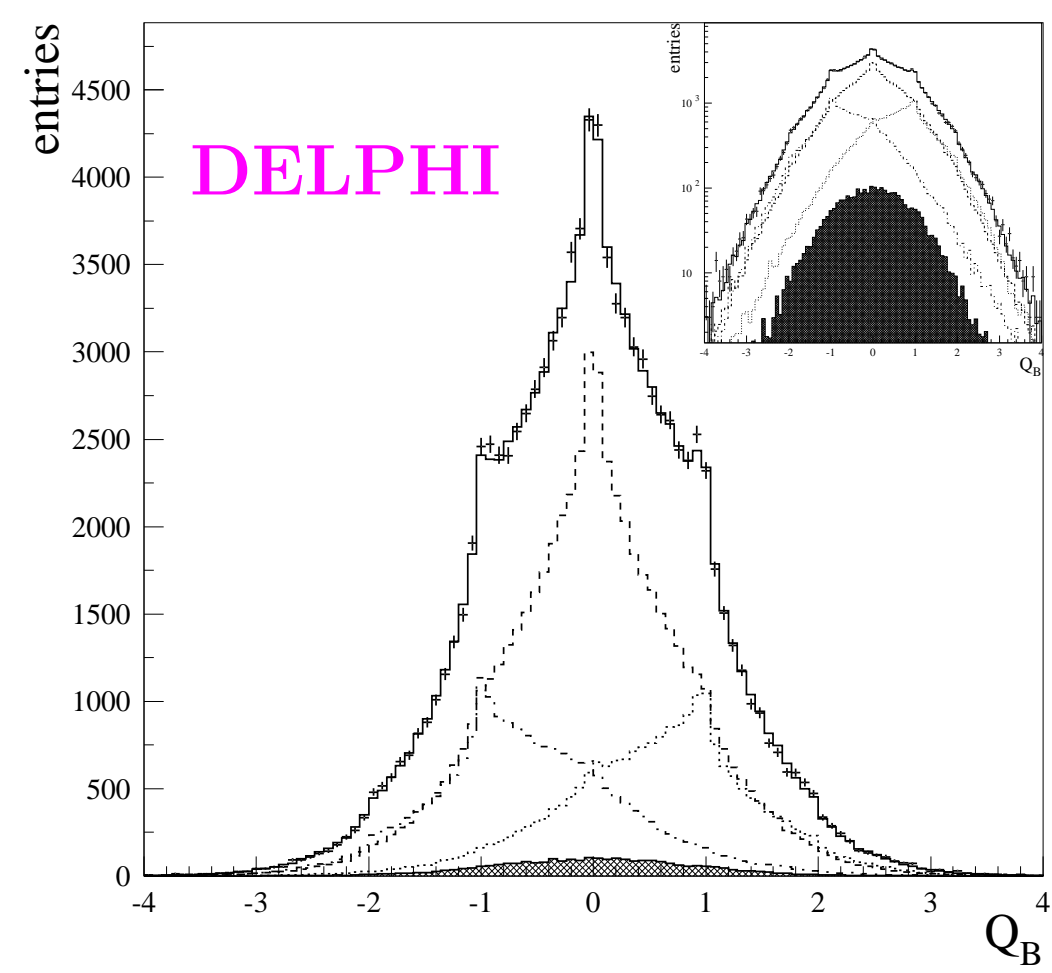

fit of the secondary vertex charge:

$$
f^{+}=(42.09 \pm 0.82 \pm 0.89) \%
$$

using $f\left(b \rightarrow \Xi_{b}^{-}\right)=(1.1 \pm 0.5) \%$

from LEP measurements of $\Xi_{b}^{-} \rightarrow \Xi^{-} l^{-} \bar{\nu}_{l} X$ and neglecting $\Omega_{b}^{-}$

$$
f_{u}=(40.99 \pm 0.82 \pm 1.11) \%
$$

HFAG from $B_{s}^{0} \rightarrow D_{s}^{-} l^{+} \nu_{l} X, \Lambda_{b}^{0} \rightarrow \Lambda_{c}^{+} l^{-} \bar{\nu}_{l} X, \Xi_{b}^{-} \rightarrow \Xi^{-} l^{-} \bar{\nu}_{l} X$

using $f_{u}=f_{d}$ and $f_{u}+f_{d}+f_{s}+f_{\text {baryon }}=1$ :

$f_{u}=f_{d}=(40.3 \pm 1.1) \%$

using time-integrated mixing

$f_{s}=(8.8 \pm 2.1) \%$ probabilities $\left(f_{d}\right.$ and $\left.f_{s}\right) \Longrightarrow$

$$
f_{u}=f_{d}=(39.7 \pm 1.1) \%
$$

$f_{\text {baryon }}=(10.7 \pm 1.8) \%$

$f_{s}=(10.7 \pm 1.1) \%$

$f_{\text {baryon }}=(9.9 \pm 1.7) \%$ 


\section{$b$ fragmentation ratios}

a) $R_{u / d}=f_{u} / f_{d} \equiv 1 \quad$ by construction (agrees with DELPHI's $f_{u}$ measurement)

b) $\gamma_{s}=\frac{2 f_{s}}{f_{u}+f_{d}}=0.27 \pm 0.03 \quad B_{s}$ production suppressed by factor $\approx 3.7$

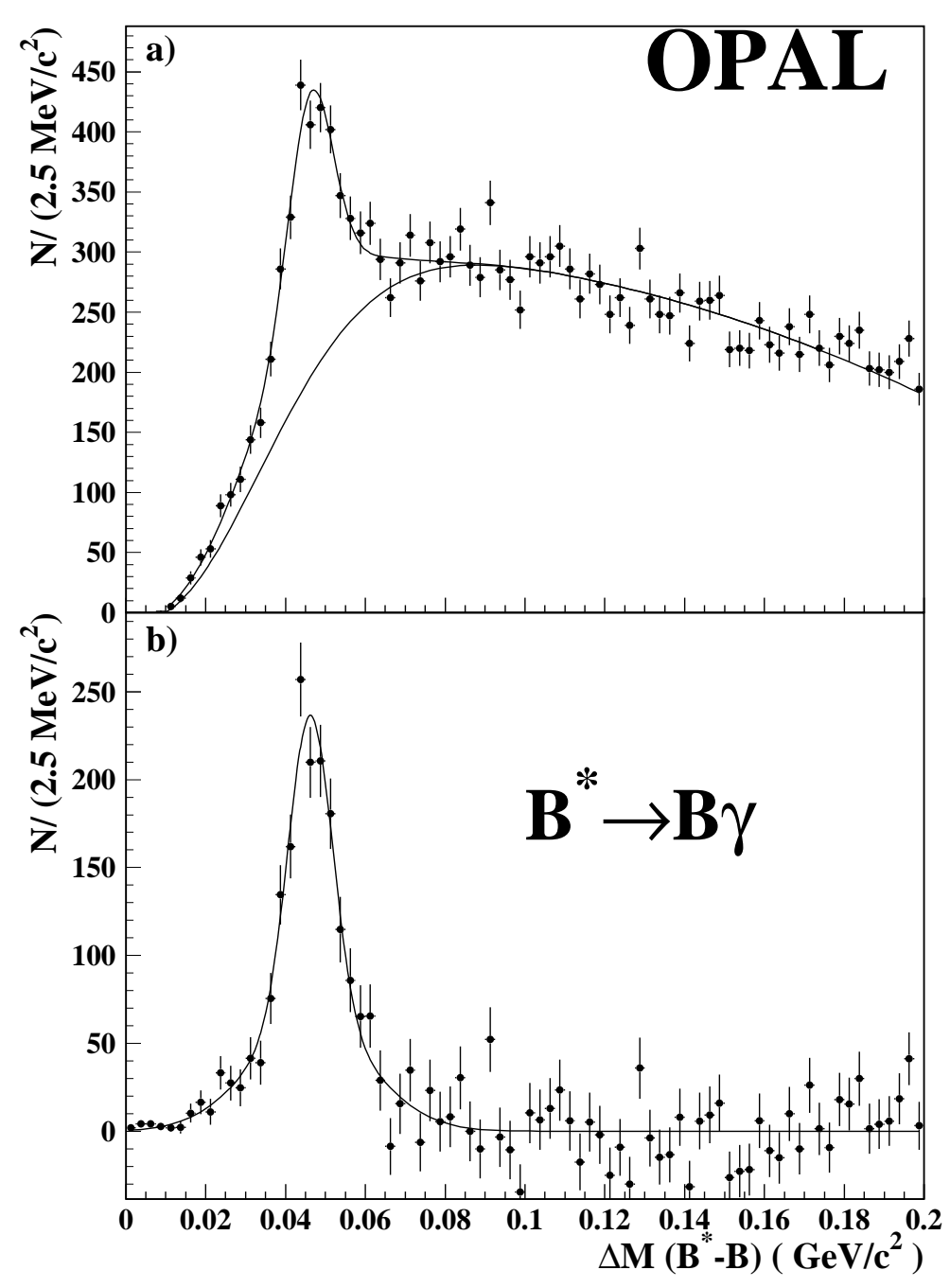

c) $P_{\mathrm{v}}$

$$
\begin{aligned}
& B^{*} \rightarrow B \gamma \\
& P_{\mathrm{v}}=\sigma\left(B^{*}\right) / \sigma(B)
\end{aligned}
$$

OPAL, ALEPH, DELPHI, L3 :

$P_{\mathrm{v}}=0.75 \pm 0.04$

$\underline{\text { agrees with spin counting }}$ 


\section{$c$ fragmentation function, NLO with Peterson FF}
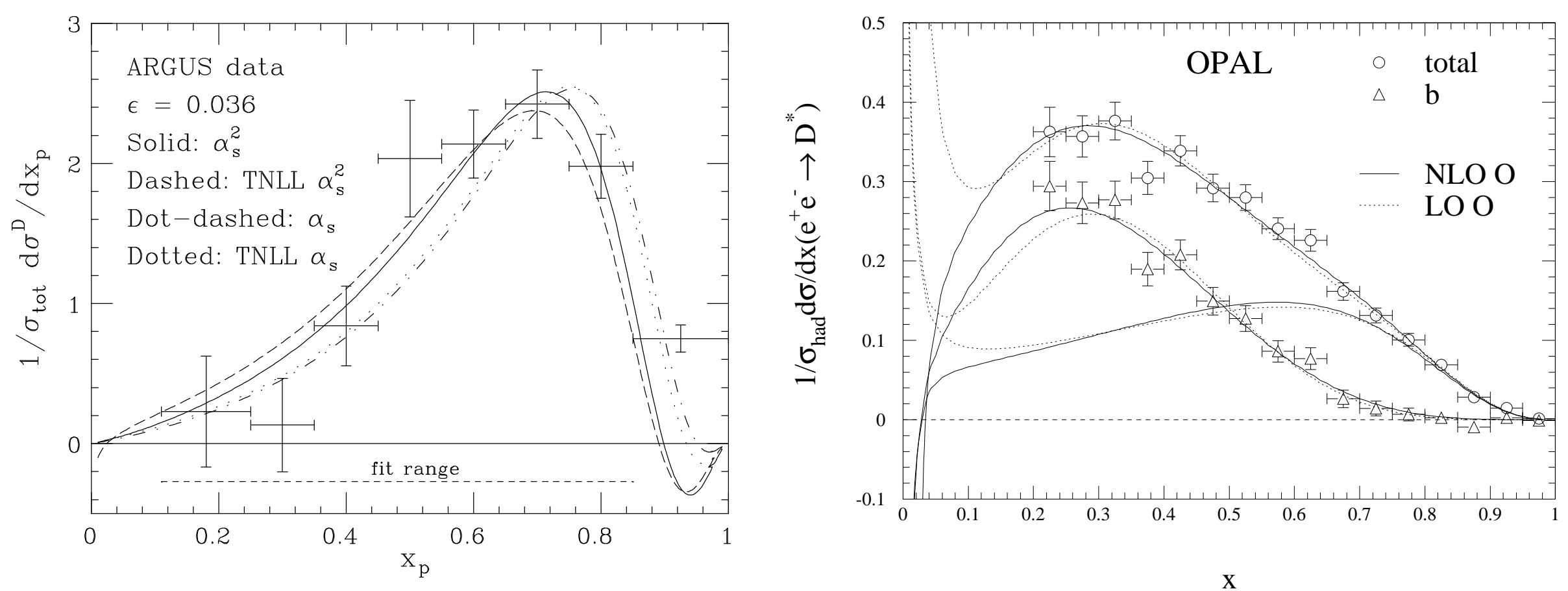

Fixed-order approach (NLO fits of P. Nason and C. Oleari) :

$$
\epsilon\left(D^{*}, D_{s}\right)=0.035 \quad \text { ARGUS data } \Leftarrow \text { Recommended } !
$$

Resummed approach ( LEP I data fit ):

Kniehl et al. $\quad \epsilon\left(D^{*}\right)=0.116$

(fit results depend from the perturbative core) 


\section{$c$ fragmentation function with LL MC}

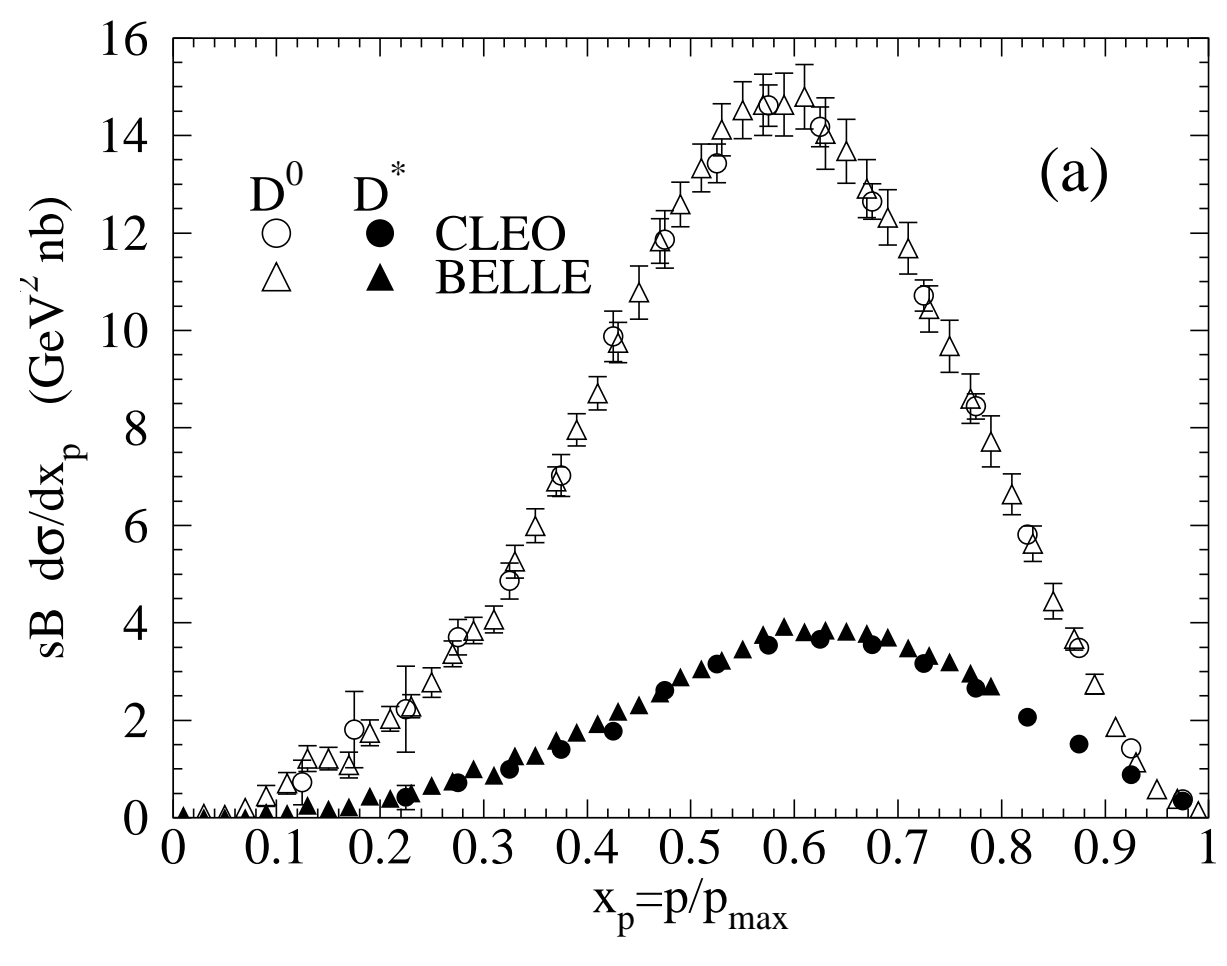

$\underline{\text { FF }\left(B e l l e, D^{*+}\right) \text { with LL Monte Carlo }}$

Bowler

$$
\text { : } \chi^{2} / N D F=541.8 / 55
$$

Lund

$$
\text { : } \chi^{2} / N D F=965.6 / 55
$$

Kartvelishvili : $\chi^{2} / N D F=1271.1 / 54$

Collins-Spiller : $\chi^{2} / N D F=1540.7 / 54$

Peterson

$$
\text { : } \chi^{2} / N D F=3003.0 / 54
$$

Recent precise measurements

\author{
from CLEO and Belle
}

$<x_{p}>=0.611 \pm 0.007 \pm 0.004\left(\mathbf{C L E O}, D^{*+}\right)$
Qualitatively, the same picture as for $b$ FF with LL MC 


\section{differential fragmentation ratios (Belle)}
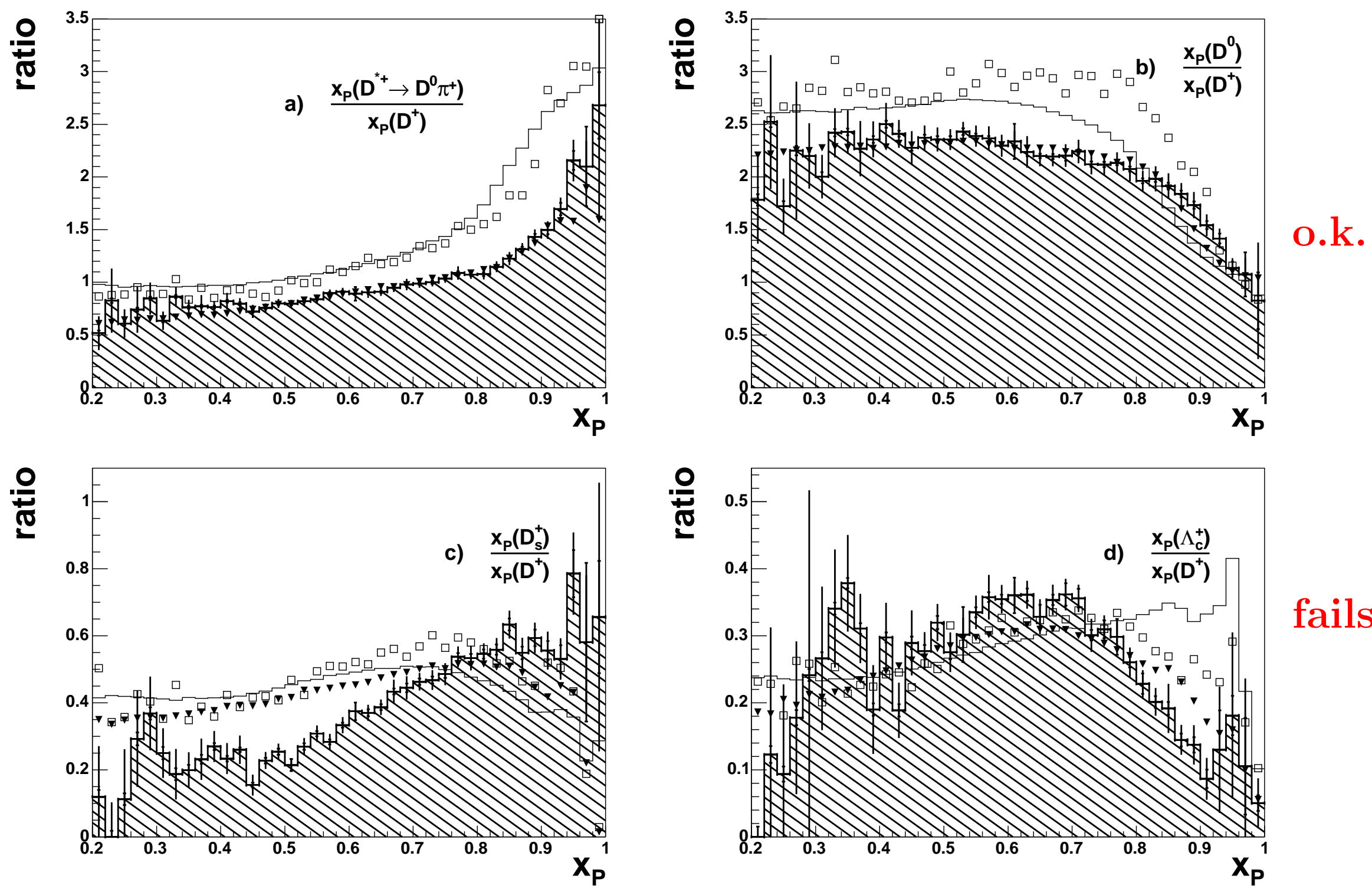

fails

hatched histograms: data

full triangles: tuned Bowler with $\operatorname{PARP}(13)=0.59\left(P_{\mathrm{v}}\right)$ 


\section{c FF with NLO+NLL+Sudakov pQCD}

Cacciari, Nason, Oleari
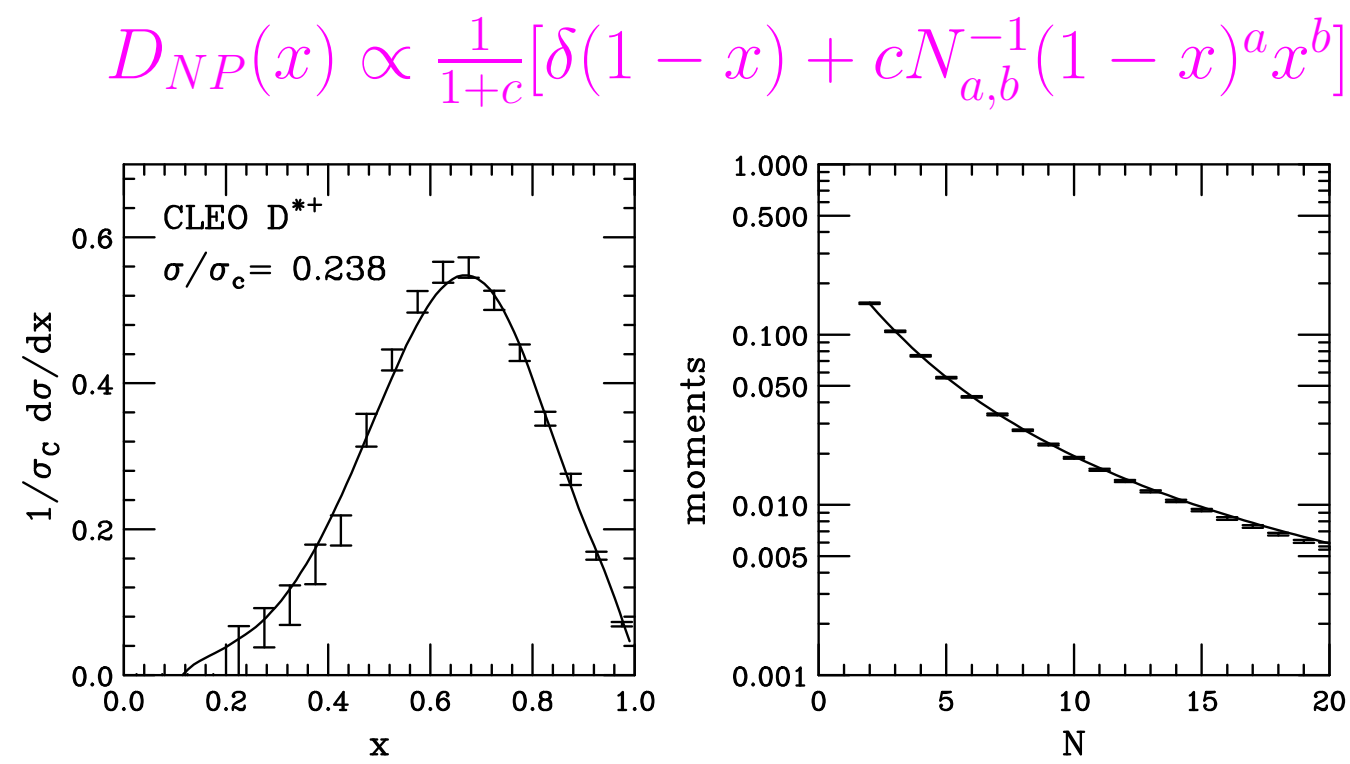

CLEO, Belle described with

$a=1.8 \pm 0.2, b=11.3 \pm 0.6, c=2.46 \pm 0.07$
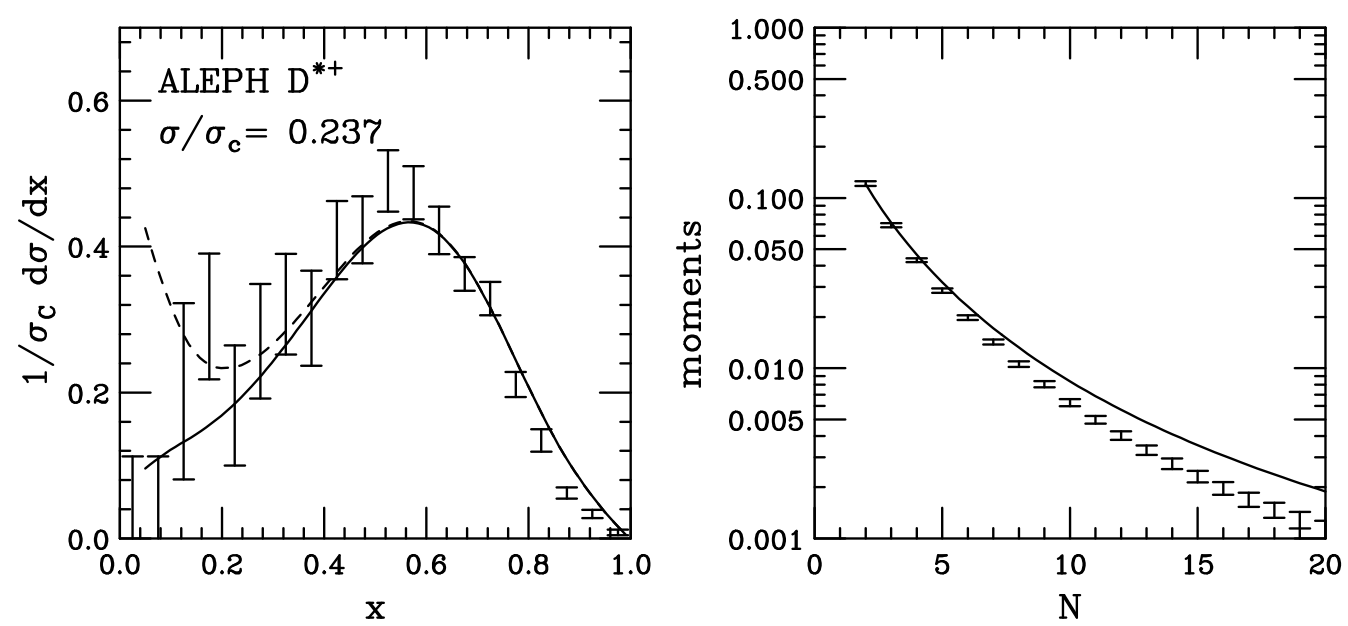

above fit does not describe ALEPH

fit to ALEPH yields

$a=2.4 \pm 1.2, b=13.9 \pm 5.7, c=5.9 \pm 1.7$

the difference for $D^{*+}$ hadroproduction up to $20 \%$

experimental info on fragmentation in hadroproduction ? 


\section{Measurement of $c \rightarrow D^{*+}$ fragmentation function}

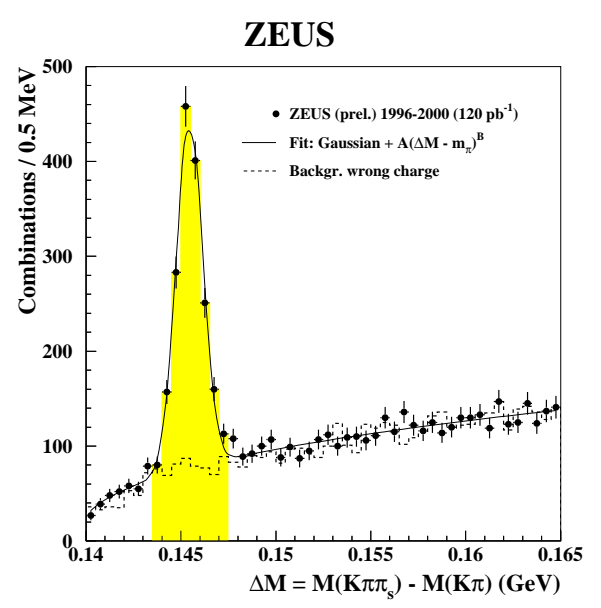

$$
\begin{aligned}
& \mathcal{L}_{\text {int }}=120 \mathbf{p b}^{-1} \\
& N\left(D^{* \pm}\right)=1268 \pm 52
\end{aligned}
$$

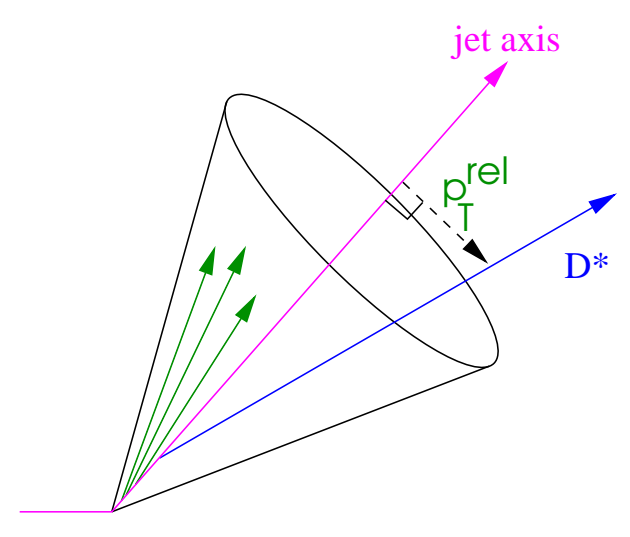

In $e^{+} e^{-}$annihilations, $D^{* \pm}$ energy is related to $\sqrt{s} / 2$. In ep ?

1) ZEUS: find jet containing $D^{* \pm}$ and relate the $D^{* \pm}$ energy to the energy of this jet: $Q^{2}<1 \mathrm{GeV}^{2}, P_{T}\left(D^{* \pm}\right)>2 \mathrm{GeV}, E_{T}^{\text {jet }}>9 \mathrm{GeV}$

$$
z=\left(E+p_{\|}\right)^{D^{*}} /\left(E+p_{\|}\right)^{\text {jet }} \equiv\left(E+p_{\|}\right)^{D^{*}} / 2 E^{\text {jet }}
$$

2) H1, jet method: $Q^{2}>2 \mathrm{GeV}^{2}, P_{T}\left(D^{* \pm}\right)>1.5 \mathrm{GeV}, E_{T}^{\text {jet }}>3 \mathrm{GeV}$

$$
z_{\text {jet }}=\left(E+p_{\|}\right)^{D^{*}} /(E+p)^{\text {jet }} \text { in } \gamma^{*} p
$$

3) H1, hemisphere method:

$$
z_{\text {hem }}=\left(E+p_{\|}\right)^{D^{*}} / \sum_{\text {hem }}(E+p) \text { in } \gamma^{*} p
$$

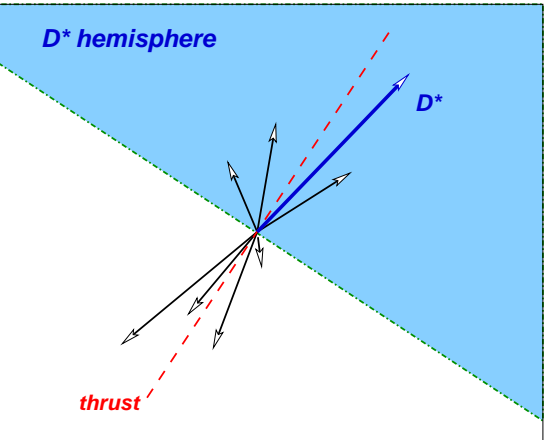




\section{Bowler and Kartvelishvili parameterizations}

Parameters are extracted using MC (PythiA or RAPGAP+PyThiA), i.e. they are optimized input parameters of the MC simulations
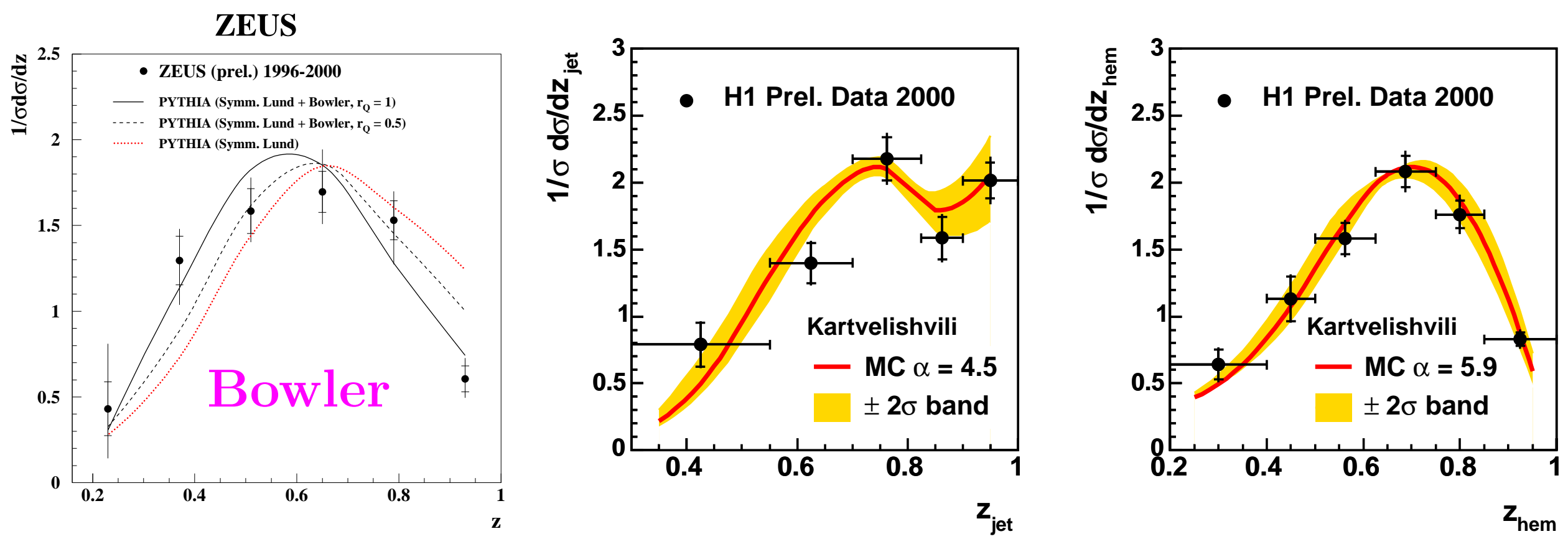

$\frac{1}{z^{1+r_{Q} b m_{Q}^{2}}}(1-z)^{a} \exp \left(\frac{-b m_{\perp}^{2}}{z}\right)$

$f(z) \propto z^{\alpha}(1-z)$

$r_{Q}=1$ (default) is preferable

$$
\alpha=4.5 \pm 0.5 \quad \text { (H1 jet method })
$$

$$
\alpha=5.9_{-0.6}^{+0.9} \text { (H1 hem. method) }
$$

$4.0<\alpha<6.8$ (H1 prel.) 


\section{Peterson parameterization: $f(z) \propto \frac{1}{z(1-1 / z-\epsilon /(1-z))^{2}}$}
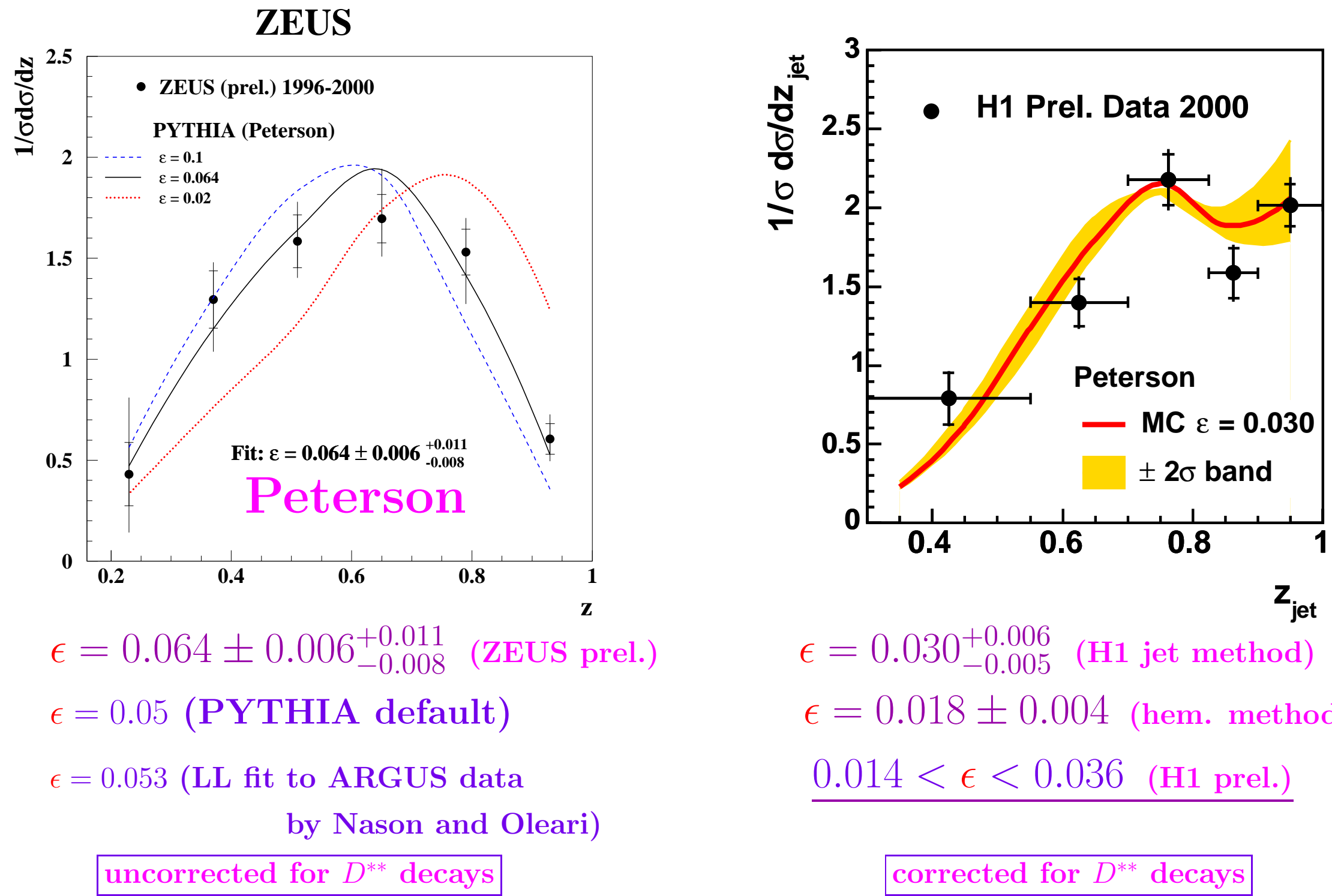

$$
\begin{aligned}
& \epsilon=0.030_{-0.005}^{+0.006} \quad(\mathrm{H} 1 \text { jet method }) \\
& \epsilon=0.018 \pm 0.004 \text { (hem. method) } \\
& 0.014<\epsilon<0.036 \text { (H1 prel.) }
\end{aligned}
$$

corrected for $D^{* *}$ decays

NLO fits are expected 


\section{Charm fragmentation function in $e p$ and $e^{+} e^{-}$collisions}

\section{ZEUS}

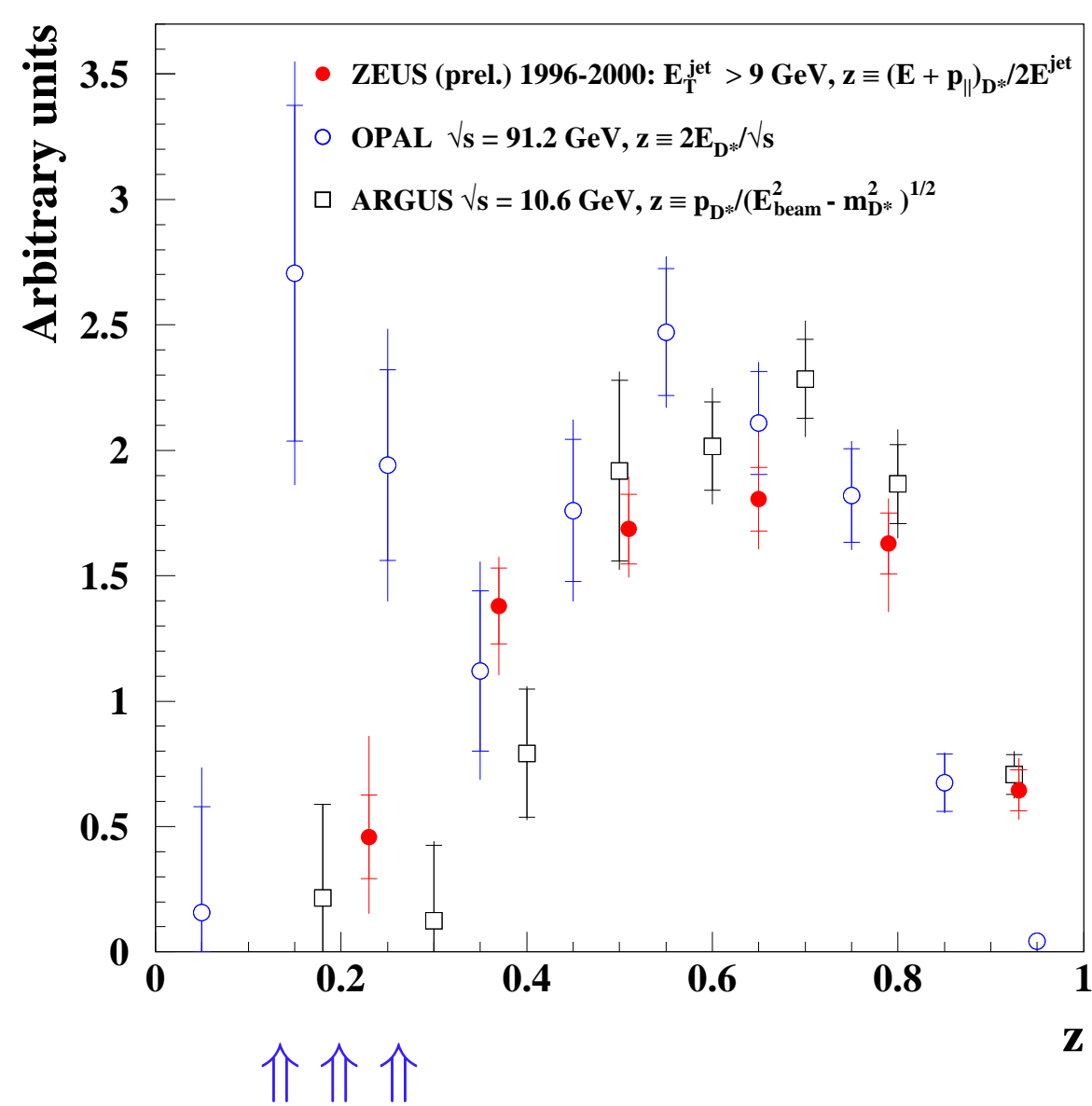

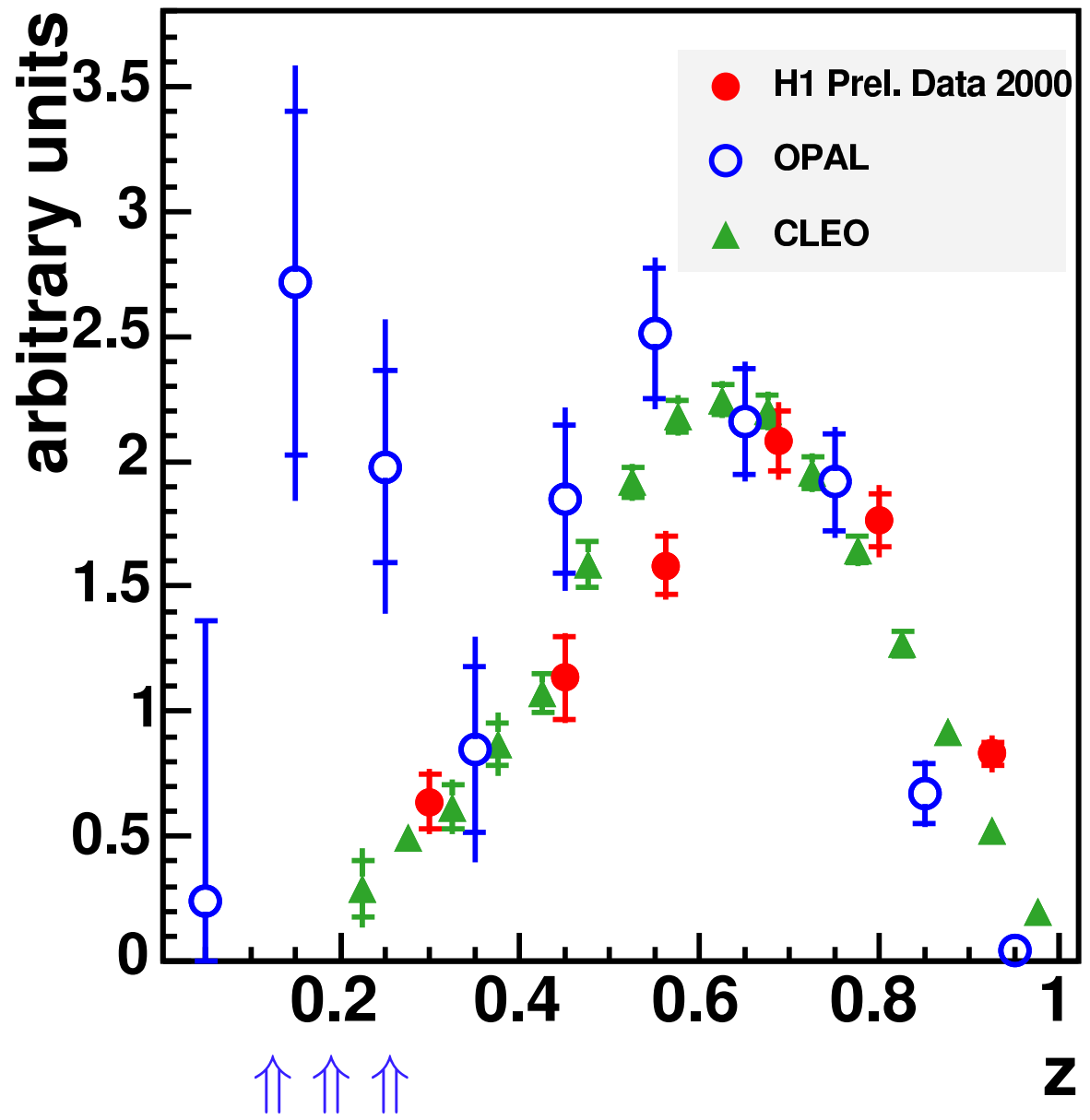

no gluon-splitting component in low-energy data

qualitative agreement 


\section{Measurement of $c$-fragmentation ratios and branchings $D^{* \pm}$ and $c$ ground states: $D^{0}, D_{s}^{ \pm}, D^{ \pm}$and $\Lambda_{c}^{ \pm}$}
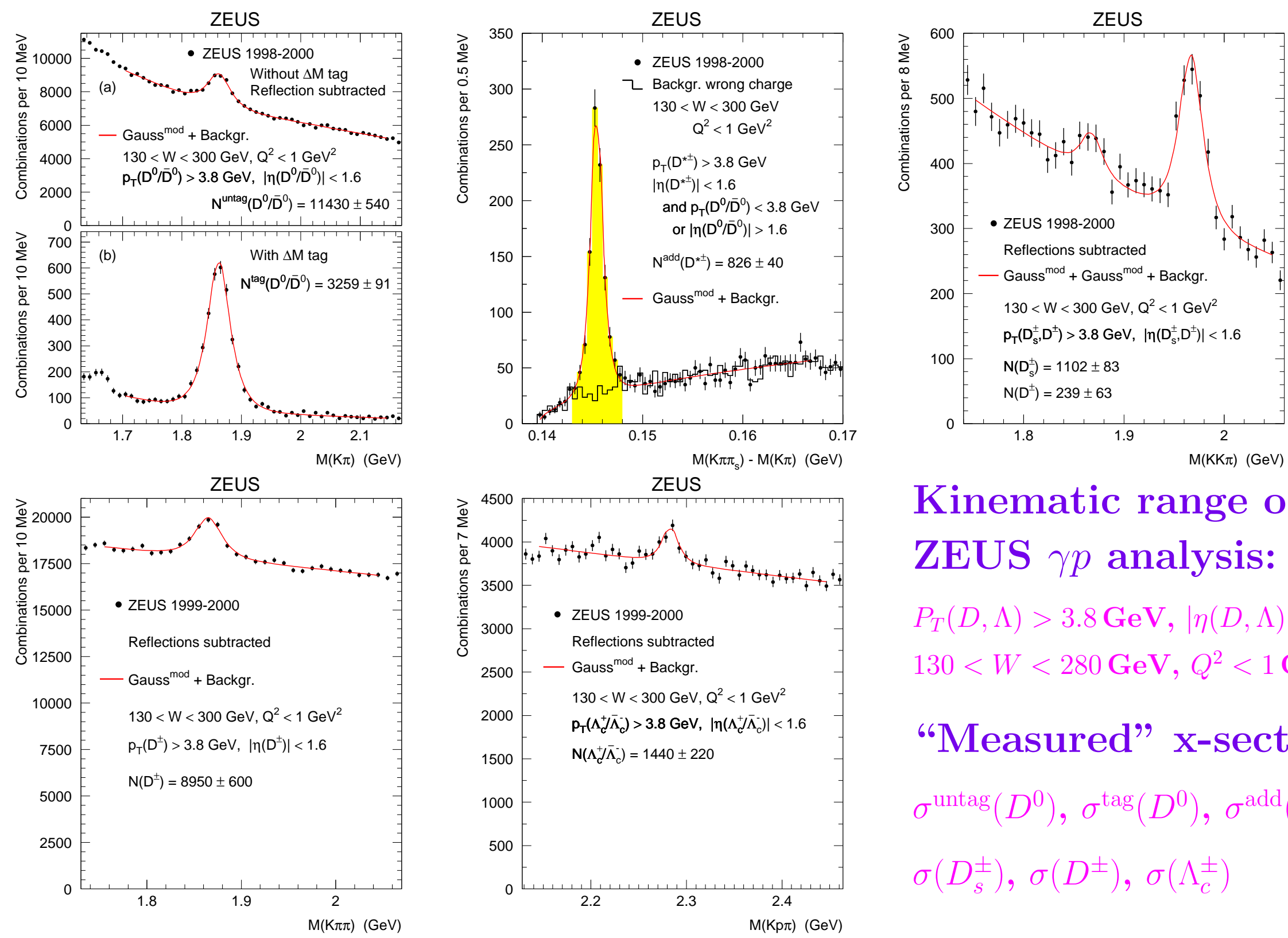

Kinematic range of

\section{ZEUS $\gamma p$ analysis:}

$P_{T}(D, \Lambda)>3.8 \mathrm{GeV},|\eta(D, \Lambda)|<1.6$

$130<W<280 \mathrm{GeV}, Q^{2}<1 \mathrm{GeV}^{2}$

"Measured" x-sections:

$\sigma^{\operatorname{untag}}\left(D^{0}\right), \sigma^{\operatorname{tag}}\left(D^{0}\right), \sigma^{\text {add }}\left(D^{* \pm}\right)$

$\sigma\left(D_{s}^{ \pm}\right), \sigma\left(D^{ \pm}\right), \sigma\left(\Lambda_{c}^{ \pm}\right)$ 


\section{$R_{u / d}$ measurement}

$$
\begin{aligned}
& R_{u / d}=\frac{c \bar{u}}{c \bar{d}}=\frac{\sigma^{\operatorname{dir}}\left(D^{0, * 0}\right)}{\sigma^{d i r}\left(D^{ \pm, * \pm}\right)}=\frac{\sigma\left(D^{0}\right)-\sigma\left(D^{* \pm}\right) \times B R}{\sigma\left(D^{ \pm}\right)-\sigma\left(D^{* \pm}\right) \times(1-B R)+\sigma\left(D^{* \pm}\right)} \\
& =\frac{\sigma\left(D^{0}\right)-\sigma\left(D^{* \pm}\right) \times B R}{\sigma\left(D^{ \pm}\right)+\sigma\left(D^{* \pm}\right) \times B R}=\frac{\sigma^{\operatorname{untag}}\left(D^{0}\right)}{\sigma\left(D^{ \pm}\right)+\sigma^{\operatorname{tag}}\left(D^{0}\right)} \quad, B R=B_{D^{*+} \rightarrow D^{0} \pi^{+}}=(67.7 \pm 0.5) \% \\
& R_{u / d}=1.100 \pm 0.078(\text { stat })_{-0.061}^{+0.038}(\text { syst })_{-0.049}^{+0.047}(\mathrm{br}) \quad(\text { ZEUS } \gamma p)
\end{aligned}
$$

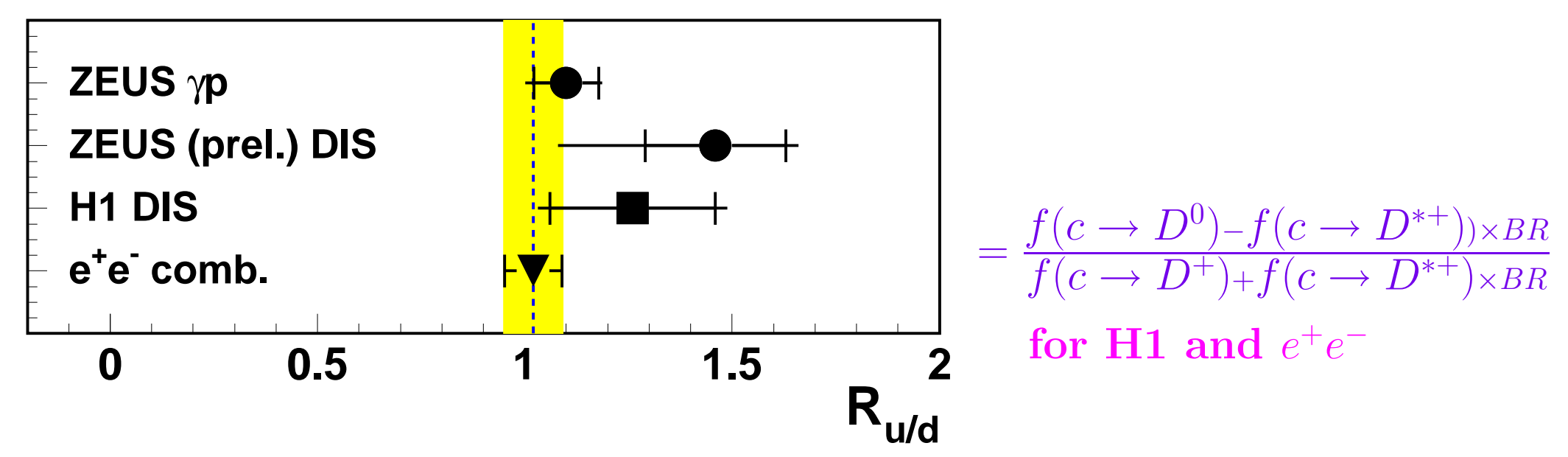

consistent with isospin invariance

$u$ and $d$ quarks are produced equally in charm fragmentation

more precise measurement in DIS ? 


\section{$\gamma_{\mathrm{s}}$ measurement}

$$
\begin{aligned}
& \gamma_{s}=\frac{2 c \bar{s}}{c \bar{d}+c \bar{u}}=\frac{2 \sigma\left(D_{s}^{ \pm}\right)}{\sigma\left(D^{ \pm}\right)+\sigma^{\operatorname{untag}}\left(D^{0}\right)+\sigma^{\operatorname{tag}}\left(D^{0}\right)+\sigma^{\text {add }}\left(D^{* \pm}\right) \cdot\left(1+R_{u / d}\right)} \\
& \left.\gamma_{\mathrm{s}}=0.257 \pm 0.024 \text { (stat) }\right)_{-0.016}^{+0.013}(\text { syst })_{-0.049}^{+0.078}(\mathrm{br}) \quad(\text { ZEUS } \gamma p)
\end{aligned}
$$

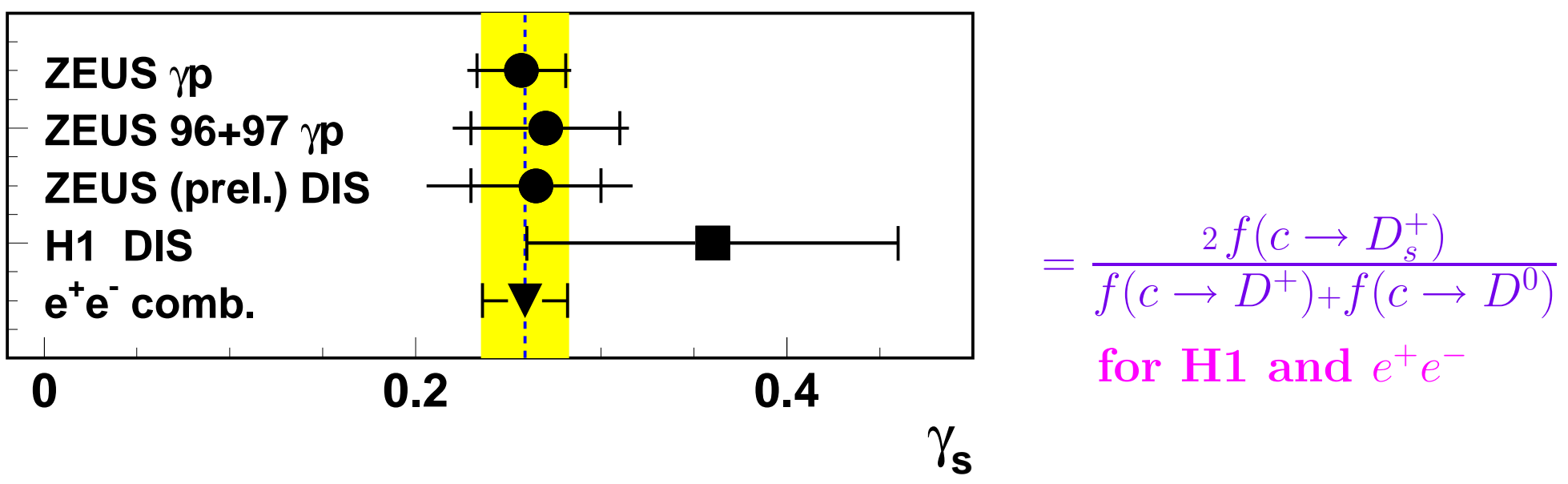

perfect agreement between measurements

\section{$D_{s}$ production suppressed by factor $\approx 3.9$ in $c$-fragmentation}

note: excited charm-strange mesons like to decay to non-strange $D$ mesons

$\Longrightarrow$ Lund strangeness-suppression parameter is $10-30 \%$ larger than the observable $\gamma_{s}$ 


$$
\begin{aligned}
& P_{\mathrm{v}}^{d} \text { measurement }\left(P_{\mathrm{v}}^{d} \equiv P_{\mathrm{v}} \text { for } c \bar{d} / \bar{c} d \text { mesons }\right) \\
& P_{\mathrm{v}}^{d}=\frac{V}{V+P S}=\frac{\sigma\left(D^{* \pm}\right)}{\sigma\left(D^{* \pm}\right)+\sigma^{d i r}\left(D^{ \pm}\right)}=\frac{\sigma^{\operatorname{tag}}\left(D^{0}\right) / B R+\sigma^{\text {add }}\left(D^{* \pm}\right)}{\sigma\left(D^{ \pm}\right)+\sigma^{\operatorname{tag}}\left(D^{0}\right)+\sigma^{\operatorname{add}}\left(D^{* \pm}\right)} \\
& P_{\mathrm{v}}^{d}=0.566 \pm 0.025 \text { (stat) }{ }_{-0.022}^{+0.007} \text { (syst) }{ }_{-0.023}^{+0.022}(\mathrm{br}) \quad(\text { ZEUS } \gamma p)
\end{aligned}
$$

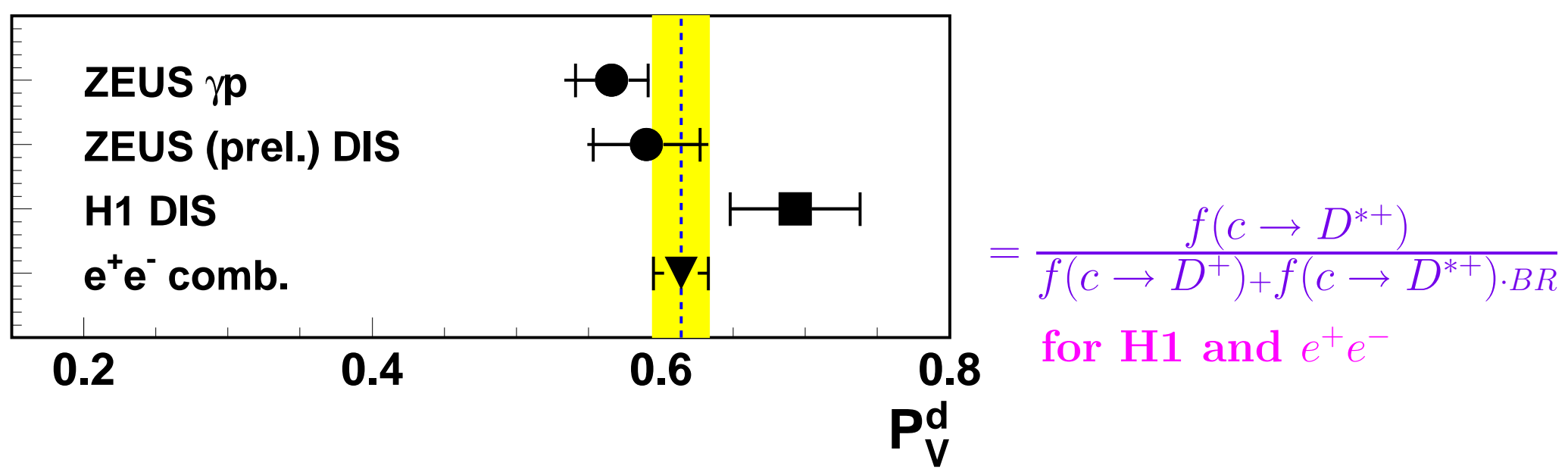

(recent precise Belle results: $P_{\mathrm{v}}^{d}=0.564$ )

$$
P_{\mathrm{v}} \neq 0.75 \Longrightarrow \text { naive spin counting does not work for charm }
$$

challenge for fragmentation models:

thermodynamics and string fragmentation predict $2 / 3$

BKL predicts $\approx 0.6$ for $e^{+} e^{-}$where only fragmentation diagrams contribute for ZEUS $\gamma p$ kinematic range, BKL prediction is $\approx 0.66$ 
Charm fragmentation branchings, $f\left(c \rightarrow D, \Lambda_{c}\right)=\sigma\left(D, \Lambda_{c}\right) / \sigma_{\mathrm{gs}}$

$$
\begin{aligned}
& \text { ZEUS } \gamma p \\
& \text { Combined } e^{+} e^{-} \text {data } \\
& f\left(c \rightarrow D^{+}\right)=0.217 \pm 0.014_{-0.005-0.016}^{+0.013+0.014} \\
& f\left(c \rightarrow D^{0}\right)=0.523 \pm 0.021_{-0.017-0.032}^{+0.018+0.022} \\
& f\left(c \rightarrow D_{s}^{+}\right)=0.095 \pm 0.008_{-0.005-0.017}^{+0.005+0.026} \\
& f\left(c \rightarrow \Lambda_{c}^{+}\right)=0.144 \pm 0.022_{-0.022}^{+0.013+0.037} \\
& f\left(c \rightarrow D^{*+}\right)=0.200 \pm 0.009_{-0.006-0.012}^{+0.008+0.008} \\
& 0.226 \pm 0.010_{-0.014}^{+0.016} \\
& 0.557 \pm 0.023_{-0.013}^{+0.014} \\
& 0.101 \pm 0.009_{-0.020}^{+0.034} \\
& 0.076 \pm 0.007_{-0.016}^{+0.027} \\
& 0.238 \pm 0.007 \pm 0.003
\end{aligned}
$$
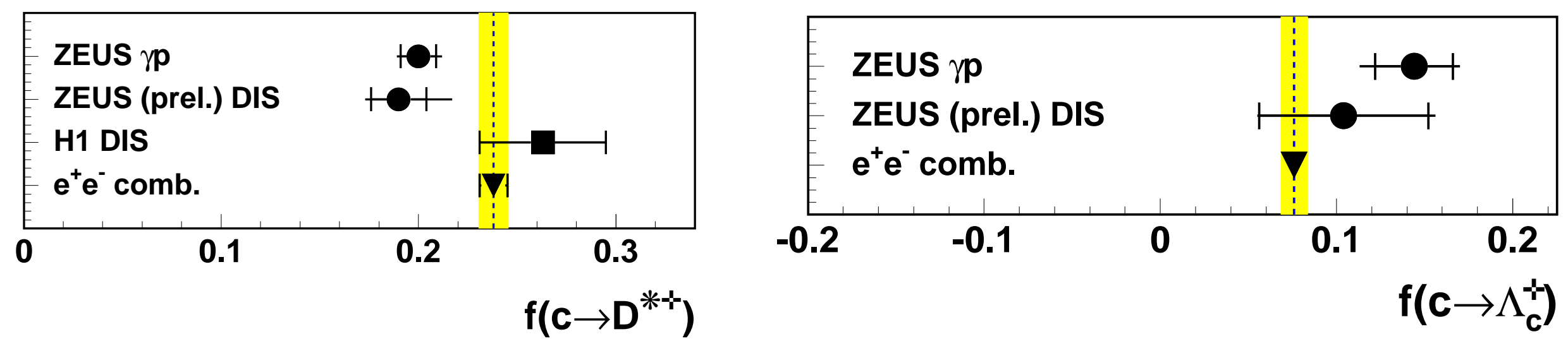

consistent with universality of charm fragmentation branchings

a half of the difference in $f\left(c \rightarrow D^{*+}\right)$ is due to the difference in $f\left(c \rightarrow \Lambda_{c}^{+}\right)$ 


\section{Summary}

Measurements of HQ fragmentation

- test pQCD calculations

- provide non-perturbative input

LL Monte Carlo with Bowler FF is generally able to describe $b / c$ fragmentation fails for $x_{p}\left(D_{s}^{+}\right) / x_{p}\left(D^{+}\right)$and $x_{p}\left(\Lambda_{c}^{+}\right) / x_{p}\left(D^{+}\right)$

NLO/NLL pQCD calculations are compatible with high-precision FF measurements

sizeable difference between fits to CLEO+Belle and ALEPH data observed

measurements of $b / c$ fragmentation ratios suggest

$u$ and $d$ quarks are produced equally in HQ fragmentation

$s$-quark production suppressed by factor $3-4$ in $c$-fragmentation

$P_{\mathrm{v}}(b)=0.75 \Longrightarrow$ naive spin counting works for beauty

$P_{\mathrm{v}}(c) \neq 0.75 \Longrightarrow$ naive spin counting does not work for charm. Why?

Measurements of charm fragmentation at HERA generally support the hypothesis that fragmentation proceeds independently of the hard sub-process 


\section{Estimates of extrapolation factors}

factors which correct the ZEUS ratios and branchings measured in the accepted $P_{T}\left(D, \Lambda_{c}\right)$ and $\eta\left(D, \Lambda_{c}\right)$ region to the full phase space

$\begin{array}{llcc} & \begin{array}{c}\text { Peterson } \\ \text { (PYTHIA) }\end{array} & \begin{array}{c}\text { Bowler } \\ \text { (PYTHIA) }\end{array} & \begin{array}{c}\text { Cluster model } \\ \text { (HERWIG) }\end{array} \\ R_{u / d} & 0.99_{-0.00}^{+0.02} & 0.99_{-0.00}^{+0.02} & 1.00_{-0.00}^{+0.01} \\ \gamma_{s} & 1.04_{-0.07}^{+0.04} & 1.00_{-0.04}^{+0.05} & 1.18_{-0.05}^{+0.07} \\ P_{\mathrm{v}}^{d} & 1.00 \pm 0.02 & 0.97_{-0.00}^{+0.01} & 0.96_{-0.01}^{+0.02} \\ f\left(c \rightarrow D^{+}\right) & 1.00_{-0.01}^{+0.02} & 1.02_{-0.02}^{+0.01} & 0.99_{-0.03}^{+0.01} \\ f\left(c \rightarrow D^{0}\right) & 0.99 \pm 0.01 & 0.98 \pm 0.01 & 0.96_{-0.02}^{+0.00} \\ f\left(c \rightarrow D_{s}^{+}\right) & 1.03_{-0.06}^{+0.03} & 1.00_{-0.03}^{+0.04} & 1.15_{-0.05}^{+0.06} \\ f\left(c \rightarrow \Lambda_{c}^{+}\right) & 1.01_{-0.05}^{+0.02} & 1.08_{-0.02}^{+0.03} & 1.46_{-0.09}^{+0.03} \\ f\left(c \rightarrow D^{*+}\right) & 1.00_{-0.03}^{+0.02} & 0.96_{-0.02}^{+0.00} & 0.93_{-0.02}^{+0.01}\end{array}$

large extrapolation factors are not expected 


\section{Fragmentation branchings for excited $D$ mesons}

Using world average for $f\left(c \rightarrow D^{*+}\right)$ :

\begin{tabular}{|c|c|c|c|}
\hline & $f\left(c \rightarrow D_{1}^{0}\right)[\%]$ & $f\left(c \rightarrow D_{2}^{* 0}\right)[\%]$ & $f\left(c \rightarrow D_{s 1}^{+}\right)[\%]$ \\
\hline \hline ZEUS (prel.) & $1.46 \pm 0.18_{-0.27}^{+0.33} \pm 0.06$ & $2.00 \pm 0.58_{-0.48}^{+1.40} \pm 0.41$ & $1.24 \pm 0.18_{-0.06}^{+0.08} \pm 0.14$ \\
\hline CLEO & $1.8 \pm 0.3$ & $1.9 \pm 0.3$ & \\
\hline OPAL & $2.1 \pm 0.8$ & $5.2 \pm 2.6$ & $1.6 \pm 0.4 \pm 0.3$ \\
\hline ALEPH & $1.6 \pm 0.5$ & $4.7 \pm 1.0$ & $0.94 \pm 0.22 \pm 0.07$ \\
\hline DELPHI & $1.9 \pm 0.4$ & $4.7 \pm 1.3$ & \\
\hline
\end{tabular}

1) the same amounts of excited $D$ mesons in $e^{+} e^{-}$and $e p$ data

2) situation with $f\left(c \rightarrow D_{2}^{* 0}\right)$ is not clear

3) $f\left(c \rightarrow D_{s 1}^{+}\right)$is twice as large as the expectation :

$\gamma_{s} \times f\left(c \rightarrow D_{1}^{0}\right) \approx 0.3 \times 2 \%=0.6 \%$

Why $f\left(c \rightarrow D_{s 1}^{+}\right)$is so large ?

Is it connected with its strange helicity ? 